\title{
Recurring Extracranial Internal Carotid Artery Vasospasm Detected by Intravascular Ultrasound
}

\author{
Tomohisa Dembo ${ }^{1,2}$ and Norio Tanahashi ${ }^{2}$
}

\begin{abstract}
A 24-year-old woman presented with headache and left-sided focal signs following multiple episodes of right monocular visual impairment. Magnetic resonance angiography revealed a decreased vascular image intensity due to a suspicious stenosis in the right internal carotid artery (ICA). The stenosis was not demonstrated on duplex sonography as it was beyond the field of view of the investigation. Intravascular ultrasound (IVUS) revealed that the outer vessel diameter was significantly reduced during stenosis, supporting the presence of vasospasm. Idiopathic recurrent extracranial ICA vasospasm was diagnosed. Recurrent vasospasms of extracranial ICA may be a distinct entity that can cause ischemic stroke.
\end{abstract}

Key words: vasospasm, cerebral ischemia, internal carotid artery, intravascular ultrasound

(Intern Med 51: 1249-1253, 2012)

(DOI: 10.2169/internalmedicine.51.7066)

\section{Introduction}

Vasospasm of the intracranial arteries is a well-known cause of cerebral ischemia in cases of subarachnoidal hemorrhage. Intracranial artery vasospasm has also been reported in isolated cases of migraine, vasculitis, and eclampsia (1). Extracranial vasospasms are rarely reported, and can be provoked by mechanical manipulations during operative interventions, vasopuncture, catheter examinations, or by drugs (such as in cases of ergot poisoning) (2). Only a few cases of spontaneous extracranial vasospasms of the internal carotid arteries have been described (1-6). Presented is a case of cerebral infarction due to idiopathic reversible vasospasm of the extracranial internal carotid artery (ICA), which was confirmed by intravascular ultrasound (IVUS).

\section{Case Report}

In March 2005, a 24-year-old right-handed woman experienced transient right visual blurring, but no headache. In July 2005, she experienced visual hazing. This was followed by a right-sided pain in her forehead and then by left-sided sensory impairment, but without paretic events, nausea, or vomiting. She took an analgesic (acetaminophen) and the symptoms resolved within 12 hours. The following day, she visited another outpatient clinic. Magnetic resonance imaging (MRI) of her head at that time did not reveal any significant findings.

In August 2005, she again experienced right visual blurring. The following day, she developed a right-sided pain from the retro-orbita to the forehead followed by numbness on the left side. One hour later, she presented to the emergency department of our hospital because of sudden development of left hemiparesis. She was otherwise healthy, and there were neither vascular risk factors present nor a history of migraine-like headaches. She was not taking oral contraceptives or any other drugs, but she reported habitually smoking 20 cigarettes per day at that time. Medical history revealed neither a family history of migraine nor a family history of stroke.

On examination, she was alert, and the visual impairment and her headache had completely resolved. However, the paresis persisted, so she was immediately admitted to the hospital. Emergent magnetic resonance diffusion-weighted imaging (DWI) revealed no evidence of cerebral infarction. MRI with a fluid attenuated inversion recovery (FLAIR) sequence revealed intra-arterial signals, mainly in the right

${ }^{1}$ Department of Neurology, EIJU General Hospital, Japan and ${ }^{2}$ Department of Neurology and Cerebrovascular Medicine, Saitama Medical University International Medical Center, Japan

Received for publication December 6, 2011; Accepted for publication January 27, 2012

Correspondence to Dr. Tomohisa Dembo, tomdembo-keio@umin.ac.jp 

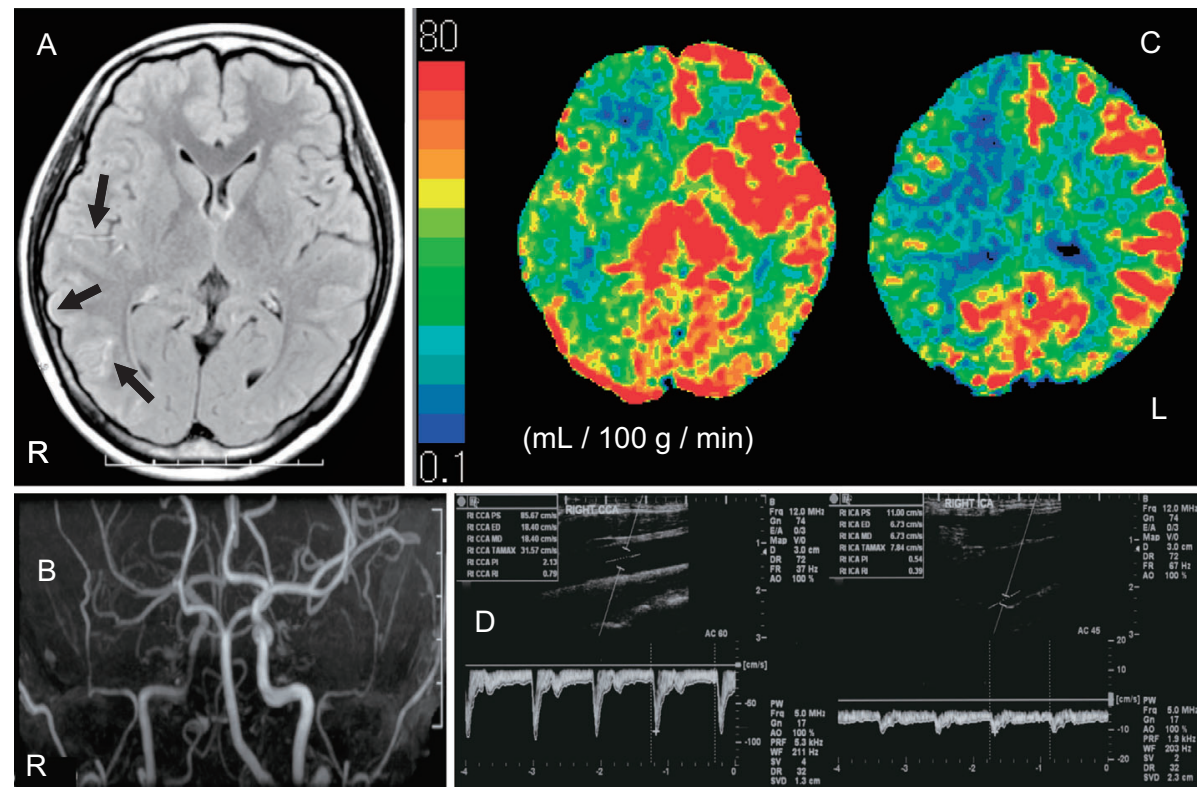

Figure 1. A: MRI with a fluid-attenuated inversion recovery sequence reveals intra-arterial signals (arrows). B: Magnetic resonance angiography reveals decreased vascular image intensity in the right internal carotid artery (ICA). C: Xenon computed tomography of cerebral blood flow reveals markedly reduced intracranial blood flow in the right ICA region. D. Pulsed-Doppler ultrasonography reveals normal findings in the right common carotid artery (left). A considerable reduction in flow rate in the right ICA is observed with the pulsed-Doppler mode (right).
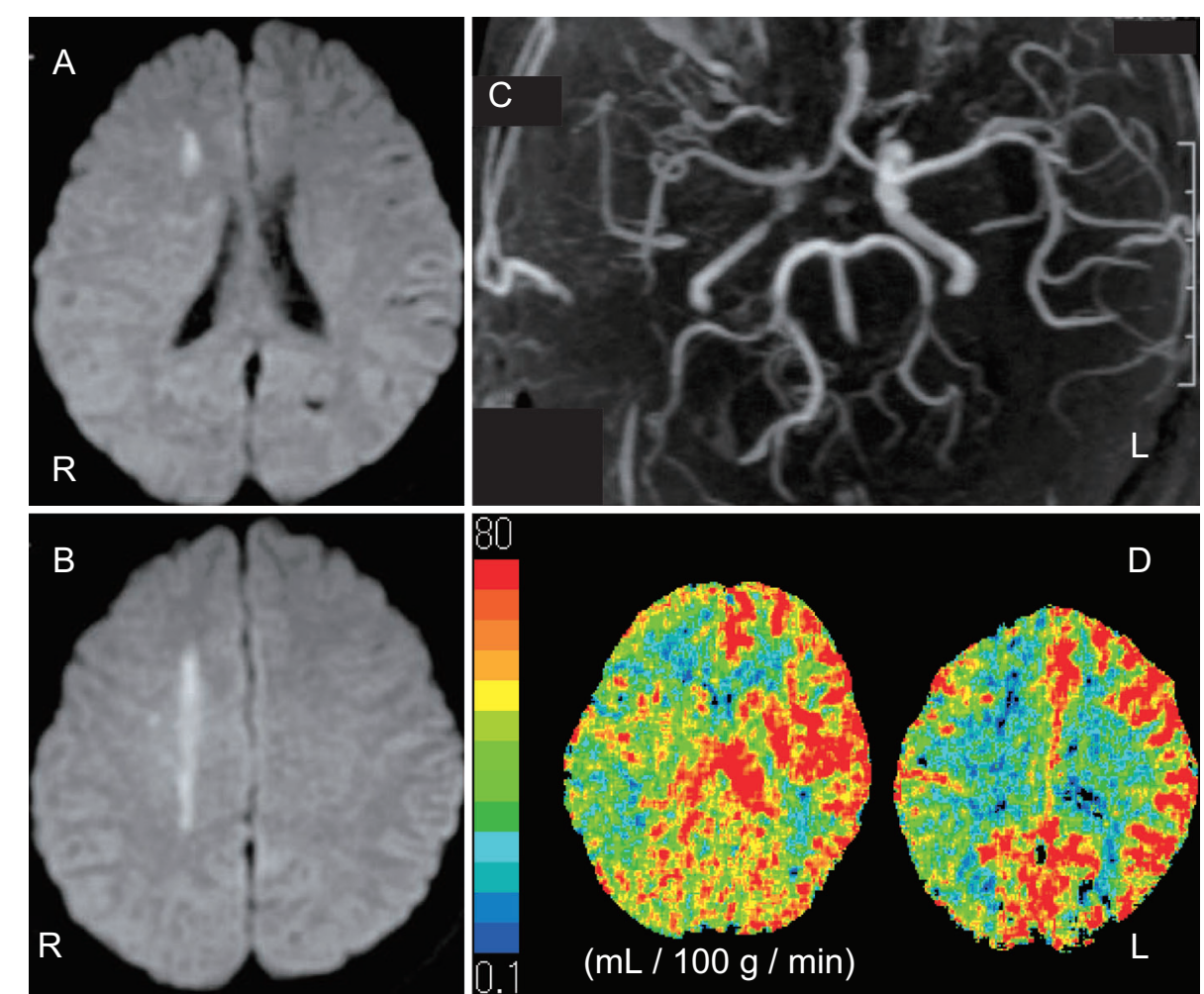

Figure 2. A, B: Magnetic resonance diffusion-weighted imaging (DWI) shows fresh cerebral infarctions in the white matter of the right frontoparietal lobe. $\mathrm{C}$ : Magnetic resonance angiography (MRA) reveals decreased vascular image intensity in the right internal carotid artery (ICA). D: Xenon computed tomography of cerebral blood flow carried out immediately after MRA shows a right ICA-region hypoperfusion. 


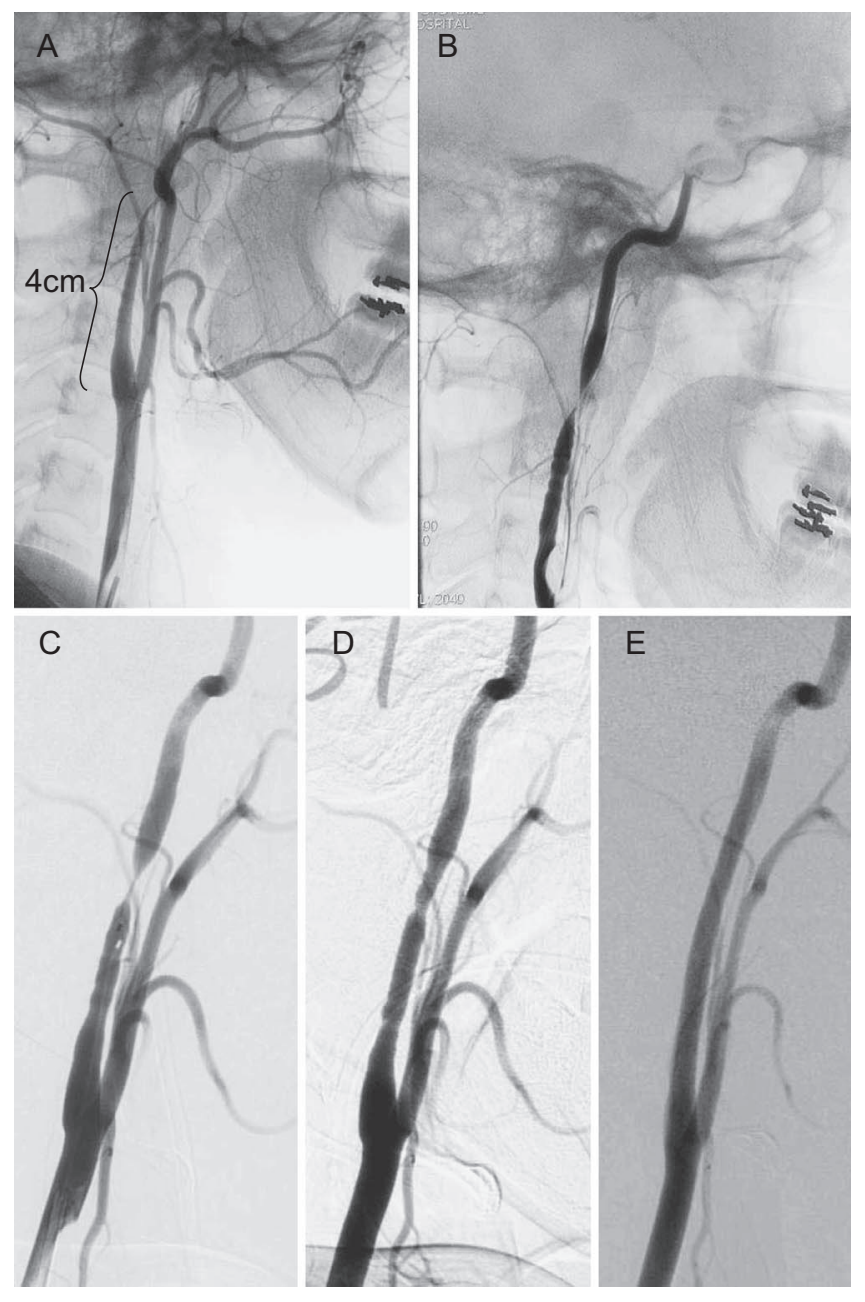

Figure 3. Digital subtraction angiography (DSA) of the right common carotid artery or the right internal carotid artery. A, B: DSA demonstrates a filiform stenosis of the right ICA about $4 \mathrm{~cm}$ above the origin of the ICA. C: DSA after local intra-arterial isosorbide dinitrate injection $(3 \mathrm{mg})$ just in front of the stenosis did not resolve the vasospasm. D: DSA after balloon angioplasty demonstrates a distinct widening of the right ICA stenosis. E. Follow-up DSA 12 days after the procedure reveals no evidence of stenosis.

middle cerebral artery (MCA) region. Magnetic resonance angiography (MRA) revealed decreased vascular image intensity due to a suspicious stenosis in the cervical segment of the right ICA. Intracranial blood flow in the right ICA region was markedly reduced, as demonstrated by a xenon computed tomography cerebral blood flow (Xe-CT CBF) examination. The stenotic lesion in the cervical segment of the right ICA was not demonstrated on duplex sonography as it was beyond the field of view of the investigation, but pulsed-Doppler ultrasound showed a reduction in the ICA flow rate (Fig. 1). Her laboratory data were negative for inflammatory disease, collagen disease, antiphospholipid antibody syndrome, coagulopathy, and pheochromocytoma. On the assumption of low-flow cerebral ischemia of hemodynamic origin due to vasospasm in the cervical segment of the right ICA, she was treated with conservative therapy. At
4 days after admission, her symptoms were completely resolved. At 8 days after admission, MRA was performed again and revealed no evidence of stenosis. On the same day, another Xe-CT CBF examination was performed and revealed normalization of $\mathrm{CBF}$ in the right ICA region. At 9 days after admission, she was prescribed a calcium antagonist. She was treated for smoking cessation as well.

Over the following 8 months, similar symptoms occurred temporarily (about six times at irregular intervals) and the symptoms regressed rapidly. In November 2005, treatment with a beta-blocker was initiated.

In May 2006, approximately 14 months after the first episode, she again experienced visual blurring and later a rightsided pain from the retro-orbita to the forehead followed by progressive left hemiparesis, and she presented to the emergency department in our hospital. Her symptoms were more strongly pronounced than in earlier episodes, and the symptoms did not improve, so she was admitted to the hospital. Emergent MRI DWI showed fresh cerebral infarctions in the white matter of the right frontoparietal lobe. MRA revealed that the vascular intensity of the right ICA was decreased. Xe-CT CBF was carried out immediately after MRA, and revealed hypoperfusion in the ICA region (Fig. 2). This finding was interpreted as a low-flow infarction of hemodynamic origin. The stenotic lesion was not demonstrated on duplex sonography as before, but pulsed-Doppler sonography showed a reduction of the flow rate in the ICA. Additionally, immediate cerebral digital subtraction angiography (DSA) was performed, which demonstrated a filiform stenosis of the right ICA about $4 \mathrm{~cm}$ above the origin of the vessel (Fig. 3). In order to make a definitive diagnosis, IVUS was performed. The IVUS catheter was pulled back from the distal to the proximal vessel, passing through the stenotic lesion. IVUS revealed no evidence of dissection or atherosclerosis. The outer vessel diameter was found to be significantly reduced during stenosis, supporting the presence of vasospasm (Fig. 4). The most likely diagnosis was idiopathic extracranial ICA vasospasm. In order to reverse ICA vasoconstriction, a local intra-arterial isosorbide dinitrate injection ( $3 \mathrm{mg}$ ) was administered just in front of the stenosis, but the vasospasm was not resolved. Balloon angioplasty was then performed, and it demonstrated a distinct widening of the right ICA stenosis (Fig. 3). The calcium antagonist and beta-blocker were discontinued, and she was treated with glycerol, fasudil hydrochloride and ozagrel sodium. MRA on the following day revealed no abnormalities. On the same day, she experienced chest oppression. Immediately, an electrocardiogram was performed, but it was normal. At 3 days after admission, the symptoms improved gradually, and at 9 days after admission, the symptoms were no longer present. At 12 days after admission, Xe-CT CBF examination revealed normalization of $\mathrm{CBF}$ in the right ICA region, DSA revealed no evidence of stenosis (Fig. 2), and contrast-enhanced MRA revealed no inflammatory changes in the vessel wall. Calcium antagonist and antiplatelet therapies were prescribed. In June 2006 she was discharged. 


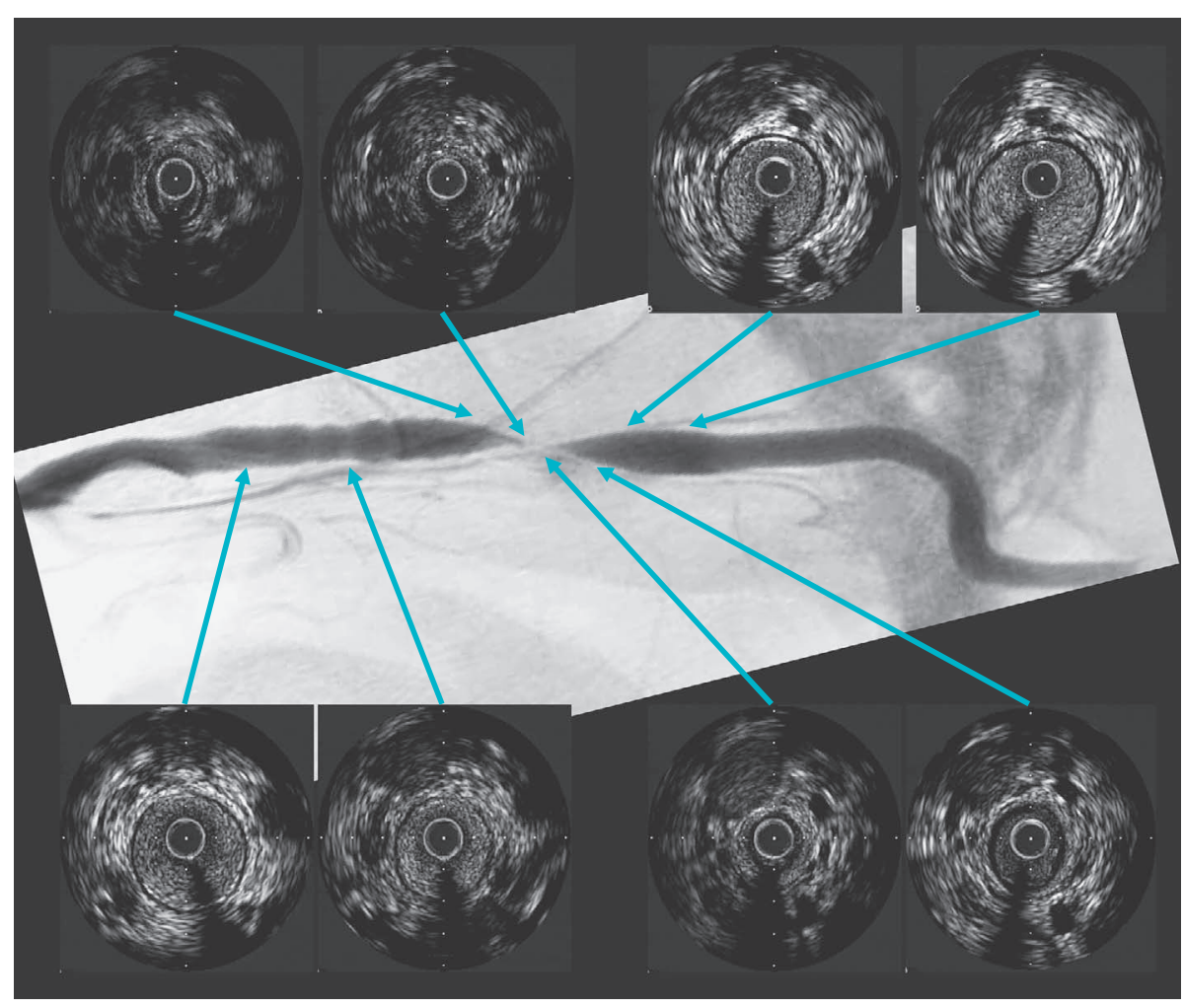

Figure 4. Intravascular ultrasound (IVUS) examination. IVUS reveals no evidence of dissection or atherosclerosis, and reveals that the outer vessel diameter was significantly reduced during the stenosis, supporting the presence of vasospasm.

\section{Discussion}

The present case report describes for the first time a patient with repeated extracranial ICA vasospasm of unknown origin with rapid and complete resolution, which was confirmed by IVUS. The distinctive features of this case are that extracranial ICA vasospasm recurred at nearly the same site, and that the patient invariably developed right visual impairment, right headache, and hemiparesis or numbness or sensory impairment on the left side in sequence.

Seven patients with spontaneous recurrent vasospasm of the cervical carotid arteries who experienced recurrent transient monocular visual impairment and cerebral infarction have previously been reported in the literature (1-6). Comparison with other reports is presented in Table 1. Despite intensive diagnostic efforts, elucidation of the definitive causes of vasospasm has been unsuccessful, and the etiology of extracranial ICA vasospasm often remains unclear. Mosso et al. discussed that migraine is not likely involved in the pathogenesis of spontaneous cervical carotid artery vasospasm (3). They came to this conclusion because their patient and only one other case (out of six cases reported) with this disorder had a history of migraine. Furthermore, the patient reported by Mosso et al. showed no temporal correlation between the occurrence of vasospasm and migraine (3). The present patient did not have a history of migraine and showed no involvement of migraine with the oc- currence of vasospasm. Furthermore, the present patient showed no temporal correlation between the occurrence of vasospasm and menstruation.

Yoshimoto et al. concluded that in young patients with ischemic stroke, extracranial ICA vasospasm could easily be confused with arterial dissection (5). However, arterial dissection was not clarified by IVUS in the present case. Furthermore, the course including the recurrence of a reversible stenosis of the left ICA at the same site supported the interpretation of these findings as vasospasm. In the present case, IVUS results enabled a definitive diagnosis of vasospasm.

Kuzumoto et al. speculated that the pathology of their case was smoking-induced vasospasm (6). In the present case, the patient also had a history of smoking; however, even after she had reportedly stopped smoking, vasospasms continued to recur. Therefore, smoking was thought to be an unlikely etiology in the present case. Arning et al. said that cervical carotid artery vasospasms could apparently occur spontaneously without a mechanical trigger (1). In the present case, the patient had no traumatic events during the preceding months and no mechanical manipulations occurred during operative interventions, vasopuncture, or catheter examinations. And mechanical compression of extracranial ICA by anatomical structures such as an elongated styloid process or stylopharyngeus muscle was not detected. Therefore, the most likely diagnosis was thought to be idiopathic extracranial ICA vasospasm.

Yoshimoto et al. stated that the distinctive feature of their 
Table 1. Comparison with Other Reports

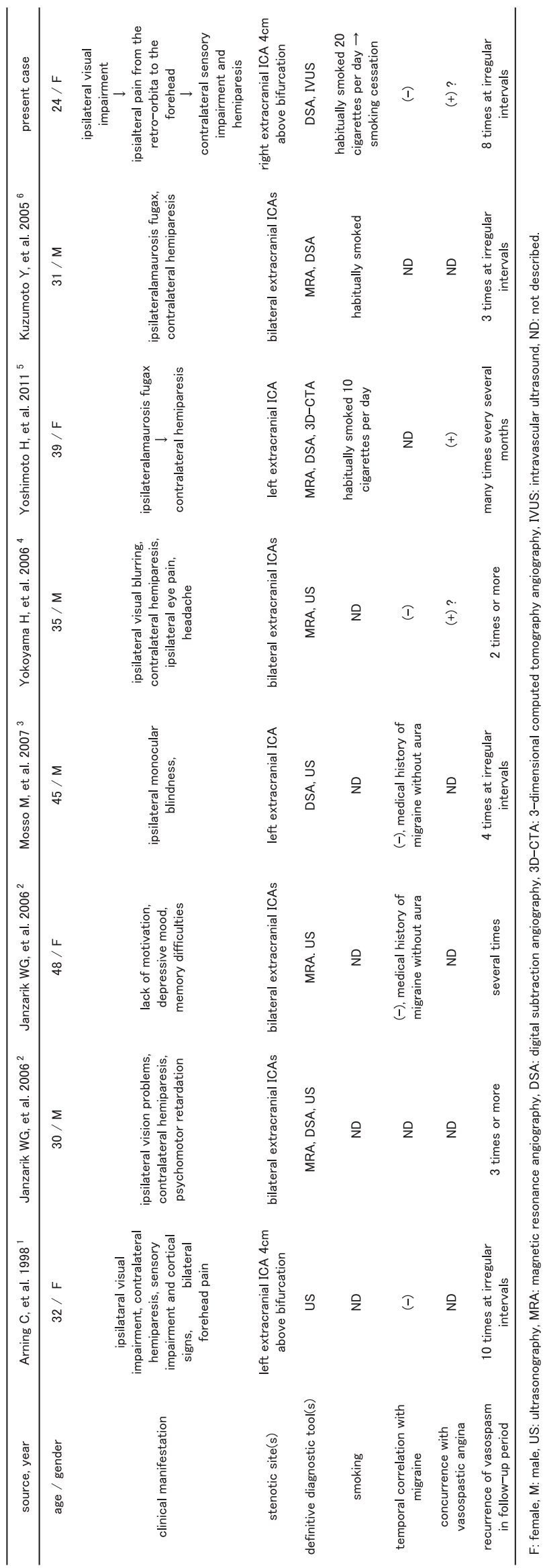

case was that extracranial ICA stenosis was accompanied by vasospastic angina (VSA) (5). The present patient experienced chest oppression, and the symptoms rapidly disappeared. This episode may support the hypothesis that VSA had occurred in the present case, but it remains unclear.

Recurrent vasospasms of extracranial ICA are a distinct entity that can cause ischemic stroke. In the present case, as with the report by Arning et al. (1), transient stenosis of the extracranial ICA was demonstrated to have occurred at nearly the same site (about $4 \mathrm{~cm}$ above the origin of the ICA), as shown by DSA, MRA, and ultrasonography. The patient developed unilateral visual impairment, a headache on the ipsilateral side, and in sequence, development of hemiparesis, numbness and sensory impairment on the contralateral side. IVUS was useful for detecting extracranial ICA vasospasm. Transient ICA vasospasm may warrant recognition as a new clinical entity, and hopefully, the pathogenesis of transient ICA vasospasm will be clarified in the future.

The authors state that they have no Conflict of Interest (COI).

\section{References}

1. Arning C, Schrattenholzer A, Lachenmayer L. Cervical carotid artery vasospasms causing cerebral ischemia: detection by immediate vascular ultrasonographic investigation. Stroke 29: 1063-1066, 1998.

2. Janzarik WG, Ringleb PA, Reinhard M, Rauer S. Recurrent extracranial carotid artery vasospasms: report of 2 cases. Stroke 37: 2170-2173, 2006.

3. Mosso M, Jung HH, Baumgartner RW. Recurrent spontaneous vasospasm of cervical carotid, ophthalmic and retinal arteries causing repeated retinal infarcts: A case report. Cerebrovasc Dis 24: 381-384, 2007.

4. Yokoyama H, Yoneda M, Abe M, et al. Internal carotid artery vasospasm syndrome: demonstration by neuroimaging. J Neurol Neurosurg Psychiatry 77: 888-889, 2006.

5. Yoshimoto H, Matsuo S, Umemoto T, Kawakami N, Moriyama T. Idiopathic carotid and coronary vasospasm: A new syndrome? J Neuroimaging 21: 273-276, 2011.

6. Kuzumoto Y, Mitsui Y, Ueda H, Kusunoki S. Vasospastic cerebral infarction induced by smoking: A case report. No To Shinkei 57: 33-36, 2005 (in Japanese, Abstract in English).

(C) 2012 The Japanese Society of Internal Medicine http://www.naika.or.jp/imindex.html 